\title{
A NEW METHOD TO DETERMINE TRABECULAR BONE ELASTIC PROPERTIES AND LOADING USING MICROMECHANICAL FINITE-ELEMENT MODELS
}

\author{
B. van Rietbergen, ${ }^{*}$ H. Weinans, ${ }^{*}$ R. Huiskes* and A. Odgaard $\dagger$ \\ *Biomechanics Section, Institute of Orthopaedics, University of Nijmegen, The Netherlands; and \\ $¥$ Biomechanics Laboratory, Orthopaedic Hospital, University of Aarhus, Denmark
}

\begin{abstract}
The apparent mechanical behavior of trabecular bone depends on properties at the tissue or trabecular level. Many investigators have attempted to determine trabecular tissue properties and loading. However, accuracy and applicability of all methods reported are limited. The small size of the trabeculae and a possible size effect are complicating factors when using traditional testing methods on single trabeculae. Other methods reported, using models that describe the trabecular structure, are of limited value because they consider bone as a repetitive structure in order to describe a reasonably large region of bone.

The present study introduces a new finite-element method strategy that enables analysis of reasonably large regions of trabecular bone in full detail. The method uses three-dimensional serial reconstruction techniques to construct a large-scale FE model, by directly converting voxels to elements. A $5 \mathrm{~mm}$ cube of trabecular bone was modeled in this way, resulting in a FE model that consists of 296,679 elements. Special strategies were developed to solve the set of equations that results from the FE approach.

Using this model in combination with experimental apparent data taken from the literature, the upper and lower boundaries for the tissue modulus were calculated to be 10.1 and $2.23 \mathrm{GPa}$, respectively. From the local stress and strain distributions it was concluded that the deformation mode of the trabeculae in the present cube was predominantly in bending. It was concluded that the method developed offers new perspectives for the study of trabecular bone.
\end{abstract}

\section{INTRODUCTION}

Trabecular bone distributes load from articular surfaces to cortical bone through its bone matrix. The trabeculae in this matrix constitute the actual loadcarrying construction. The material properties of the trabeculae, in combination with their architecture, determine the strength and stiffness of trabecular bone Under loading, these properties determine the strains at microstructural levels that are believed to regulate the biological adaptive processes in trabecular bone (Frost, 1987; Martin and Burr, 1989). In spite of their importance, little is known about the microstructural properties and loading of the individual trabeculae.

Several attempts have been described in the literature to determine the trabecular tissue material properties. In these studies a wide range of possible values was reported, with many studies suggesting that the actual tissue modulus is much less than generally accepted for cortical bone (16-20 GPa). Methods reported include traditional tensile and bending tests applied to single trabeculae or similar sized specimens. Ryan and Williams (1989) used tensile testing experiments on single trabeculae, and found a tra-

Received in final form 14 February 1994.

Corresponding address: Rik Huiskes, Biomechanics Section, Institute of Orthopaedics, University of Nijmegen, P.O. Box 9101,6500 HB Nijmegen, The Netherlands. becular tissue modulus of 0.4-3.6 GPa. Other studies, using three or four point bending experiments, have resulted in tissue values of 3.81-5.72 GPa (Choi and Goldstein, 1992; Choi et al., 1990; Kuhn et al., 1989). Recently, Rho et al. (1993) determined the Young's tissue modulus of single trabeculae, using microtensile testing and ultrasonic techniques, finding a tissue modulus of 10.4-14.8 GPa. An indirect way to determine the tissue properties uses a numerical or analytical model representing a typical piece of trabecular bone, in combination with apparent experimental data. These models can also be used to determine the stresses and strains in the trabeculae. Williams and Lewis (1982) used a two-dimensional FE model of a trabecular cross-section in combination with experimental results. They found a trabecular tissue modulus of $1.3 \mathrm{GPa}$. In later studies, three-dimensional models were used to model the trabecular structure. Gibson (1985) considered trabecular bone as a cellular material, and used different analytical cell models to represent the trabecular architecture. She found that cancellous bone develops four types of structures, dependent on its density. Beaupré and Hayes (1985) also modeled trabecular bone as a repetitive cellular structure. They used a three-dimensional FE model of the cellular structure to calculate the apparent stiffness and the tissue stress and strain distribution. Recently, Hollister et al. (1991) introduced homogenization theory in combination with the FEM for modeling large regions of trabecular bone in 
detail. This theory assumes trabecular bone to be a structure of unit cells, each having the same microstructural morphology. The unit cells can either be simplified, regular structures (Hollister et al., 1991), or represent the detail of trabecular morphology (Hollister and Kikuchi, 1992). The accuracy and applicability of all methods reported is limited. Methods using single trabeculae or similar sized machined specimens suffer from size effects or irregularities due to the cutting of the specimens. Also, these methods cannot be used to determine in situ tissue stresses and strains. The accuracy of methods that use a trabecular bone model depends on their capability to describe a realistic trabecular architecture. Two-dimensional models can only represent realistic bone morphology in those rare cases that trabecular bone has a cylindrical structure of constant cross-section. All three-dimensional microstructural models mentioned above assume trabecular bone to be built of identical unit cells. However, it is clear that such a repetitive structure is very rare for trabecular bone.

A more accurate way for determining tissue stresses and strains in a specific piece of trabecular bone can be obtained if the structure of a large region of bone can be modeled in detail. Two methods are presently available to measure and represent the trabecular structure on a microlevel. One is a nondestructive method, using micro-CT-scanning, with a typical voxel size of $50 \mu \mathrm{m}$ (Feldkamp et al., 1989). The other technique for routine three-dimensional reconstruction involves automated serial sectioning, which can provide a resolution of about $1 / 1000$ of the specimen size, i.e. $10 \mu \mathrm{m}$ for a $10 \mathrm{~mm}$ specimen. Hollister and Kikuchi (1992), Hollister et al. (1992), Fyhrie et al. (1992), Edidin et al. (1993) and Fyhrie and Hamid (1993) have used voxel data sets to construct detailed FE models of small pieces (up to $2.3 \mathrm{~mm}$ cubes) of trabecular bone. Until recently, however, efforts to represent a substantial piece of bone in a FE model, for example the size of mechanical test specimen, have failed due to restrictions in computer memory and CPU time. In order to relate local strains in bone to those on the apparent level, a bone specimen must be large enough to allow for a sensible definition of apparent (continuous) properties. Harrigan et al. (1988) have shown that this condition is met for specimens that span at least five intertrabecular lengths.

In the present study a newly developed FE strategy is introduced which does enable such a full, realistic three-dimensional analysis. The purpose of the present study was twofold. First, to demonstrate how this strategy can be used to determine the tissue modulus as a function of the apparent modulus by simulating a compressive test, similar to those reported frequently in the literature. Second, to compare local trabecular stresses and strains and other quantities, to those on the apparent level. The same full three-dimensional FEM model of a trabecular cube was used in both cases, with different boundary conditions, and the newly developed FE solution strategy was applied.

\section{METHODS}

A $7 \times 7 \times 7 \mathrm{~mm}$ trabecular bone cube was sectioned out of a proximal human tibia. The three-dimensional microstructure of this cube was obtained by digitizing 176 slices, using a resolution of $176 \times 248$ pixels (Odgaard et al., 1990). A three-dimensional reconstruction of this bone cube is shown in Fig. 1. The rectangular voxels in the digitized cross-sections were converted to equally sized eight-node brick elements using a three-dimensional FE preprocessor developed in our laboratory. To eliminate rough boundaries and to reduce the data set, only $128 \times 128 \times 128$ voxels taken from the center of the reconstruction were used to construct the FE model, representing a $5.15 \times 3.64$ $\times 5.12 \mathrm{~mm}$ cube. The connectivity of the resulting element mesh was checked for elements not connected to the main structure, also using a special-purpose program. All elements in the model are rectangular and equally sized: $40.26 \times 28.44 \times 40 \mu \mathrm{m}$ in the $x$-, $y$ and $z$-directions, respectively. The cube has 128 voxels at each side (Fig. 2), and hence (128) ${ }^{3}$ voxels in total, of which 296,679 represent bone. Accordingly, the volume fraction of the bone equals $V_{v}=296,679 /(128)^{3}=$ 0.14 . The total number of degrees of freedom equals $1,381,602$.

A special-purpose FE code was developed to solve the set of equations that results from the finite-element approach: $K u=f$, with $K$ the global stiffness matrix, $\mathbf{u}$ the displacement vector, and $\mathbf{f}$ the external forces. The conjugate gradient iterative solving technique, with diagonal scaling as a preconditioner (Hughes et al., 1987; Strang, 1986), was used to calculate the unknown displacement vector $\mathbf{u}$. Each iteration requires a multiplication of the global stiffness matrix $\mathbf{K}$ with a direction-vector $\mathbf{p}$. The memory required to solve this problem was reduced by three separate strategies. First, the entire global stiffness matrix is never actually computed. Instead, an element by element (EBE) approach (Hughes et al., 1987) is applied which allows for the multiplication at element level $K p=\sum K_{e} p_{e}$, where $K_{e}$ is the element stiffness matrix (order 24) and $\mathbf{p}_{\mathrm{e}}$ the decomposed vector p. Second, because all elements are identical in size and have the same orientation, the global structure can be described by the element connectivity alone. Hence, no nodal coordinate data have to be stored. Finally, all elements have identical material properties which allows for an identical element stiffness matrix $\mathbf{K}_{\mathrm{e}}$ for all elements. This reduction even applies when each element would have a different Young's modulus, because the isotropic element stiffness matrix is a linear function of this modulus. Compared to traditional EBE solving techniques which must store all element stiffness matrices, the required core size is drastically reduced as only one element stiffness matrix has to be stored. This can be demonstrated for the present model, where instead of 296,679 element stiffness matrices, now one element stiffness matrix has to be stored, or a reduction in memory requirements by $99.99966 \%$. 


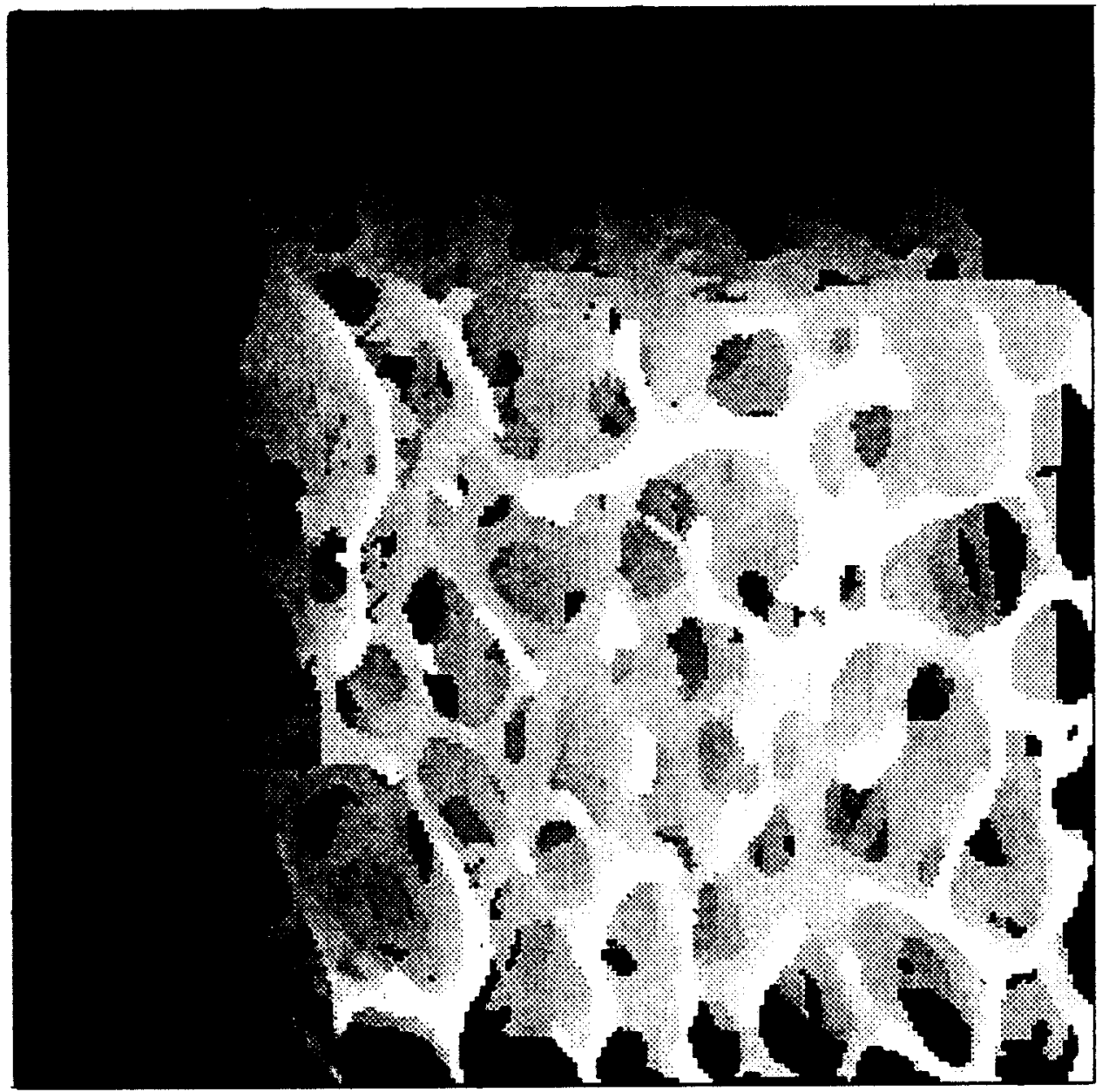

Fig. 1. The $7 \times 7 \times 7 \mathrm{~mm}$ three-dimensional reconstruction of the bone cube taken from the proximal human tibia plateau. A $5.15 \times 3.64 \times 5.12 \mathrm{~mm}$ part of it represents the FE mesh as well, since each voxel is represented by a brick element in the FE model, which has 296,679 elements in total.

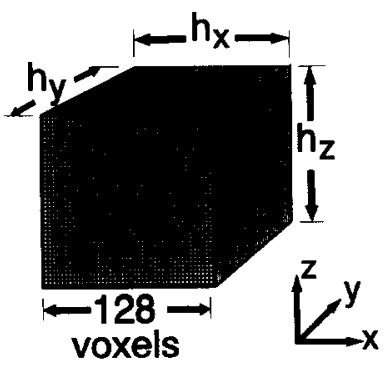

Fig. 2. Definition of the dimensions of the cube. 


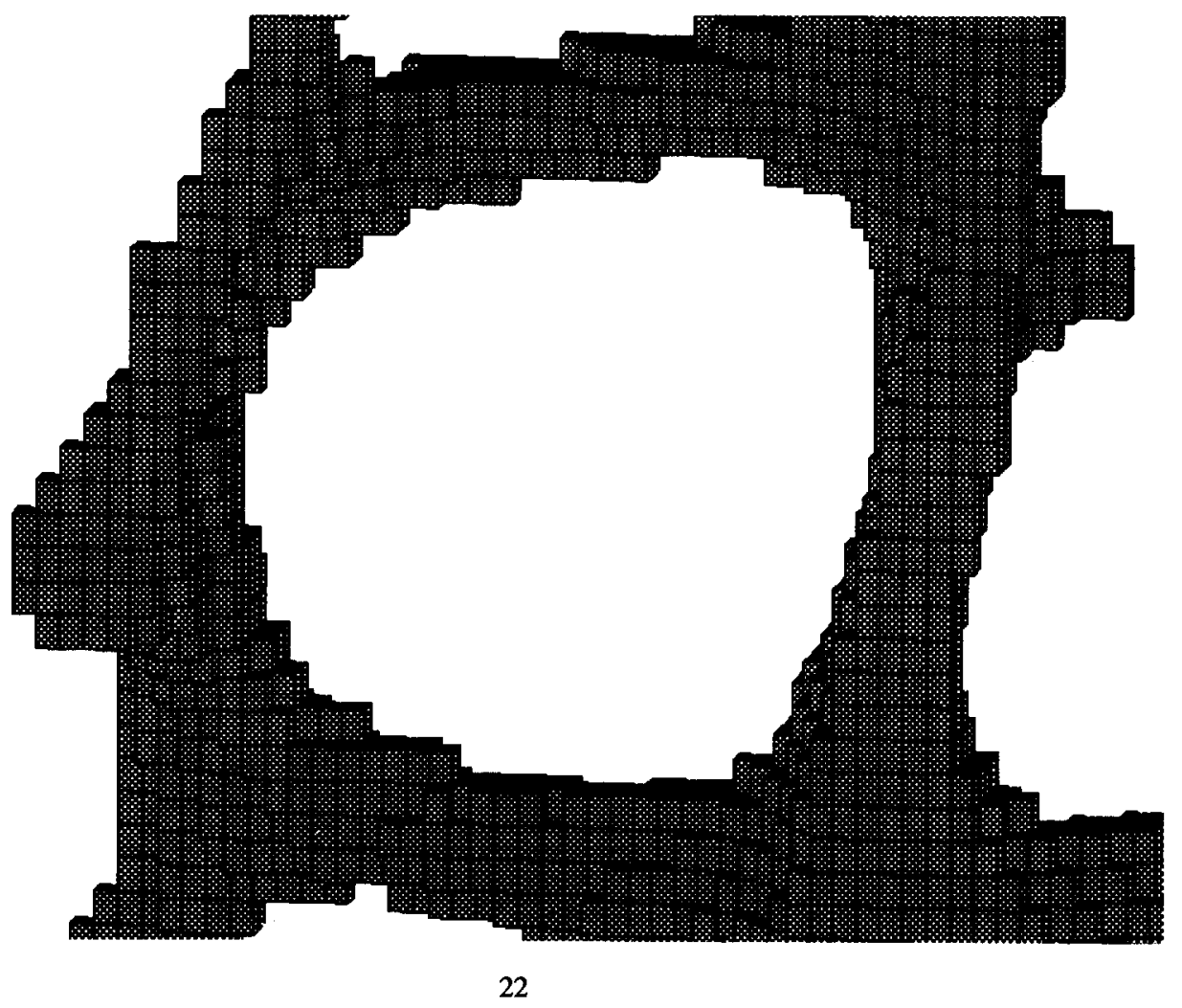

Fig. 4. Detail of the FE model, showing the element size relative to the trabecular size. 

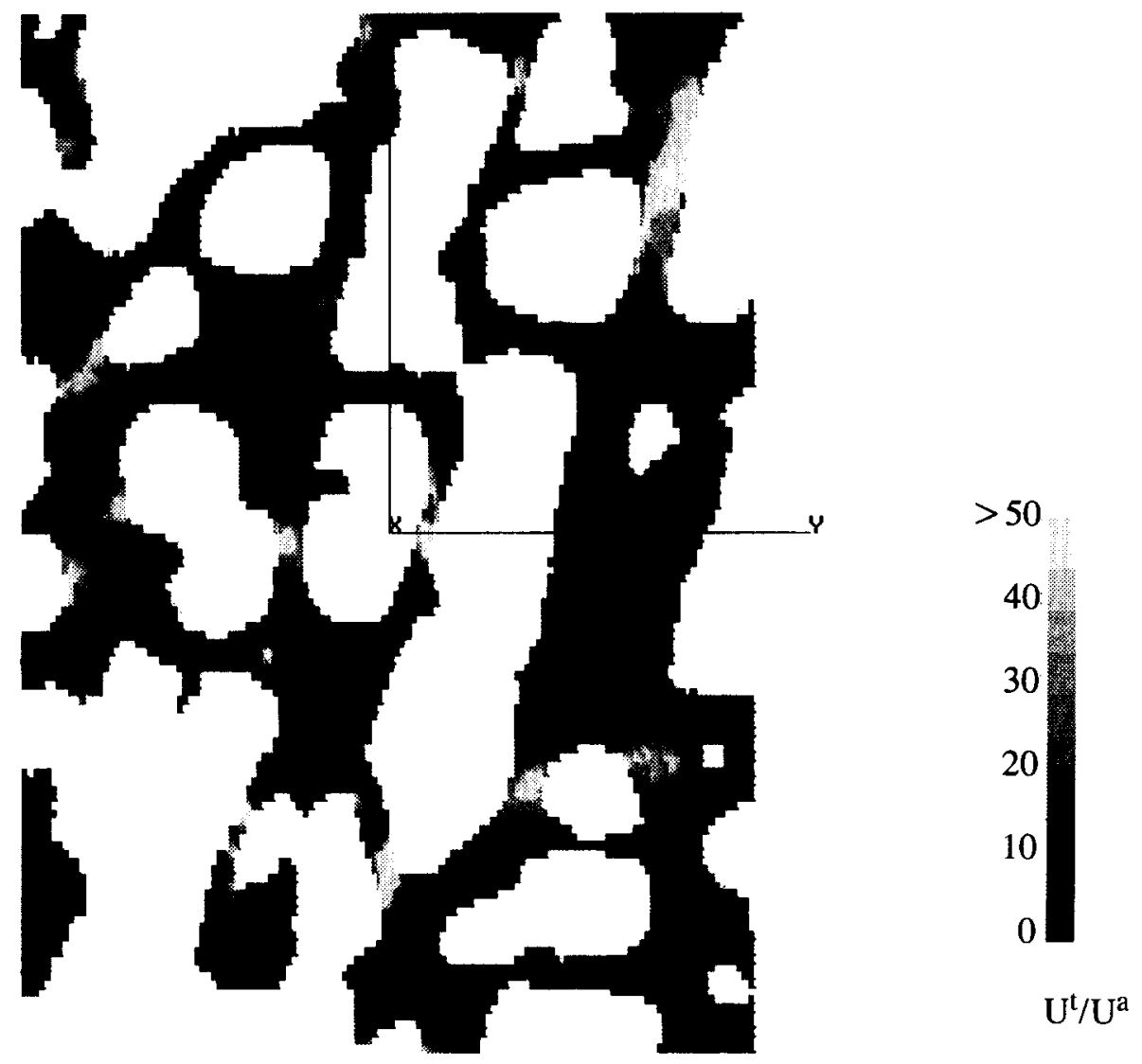

Fig. 9. Contour plot for the tissue strain energy density distribution $U^{\prime}$ in a ten-element thick cross-section taken from the middle of the cube. White areas are highly loaded $\left(U^{t}>50 U^{a}\right)$, black areas are unloaded. 
To reduce the CPU time, vectorization schemes as described by Hughes et al. (1987) and Hayes and Devloo (1986) were applied to the global stiffness matrix-vector product, taking full advantage of the CRAY-YMP computer which was used for the calculations. To monitor the convergence of the process the norm of the residual force vector $\left\|f_{\text {res }}\right\|$ was calculated every increment. The iterative process was terminated as soon as this norm was less than $1 E-6 N$. After calculating the reaction forces, the accuracy of the results was determined by calculating the ratio $\left\|f_{\text {res }}\right\| /\left\|f_{\text {reac }}\right\|$, with $\left\|f_{\text {reac }}\right\|$ the norm of the reaction force vector. The solution was considered sufficiently accurate if this ratio was less than $1 E-5$.

The validity of the algorithms developed was checked by calculating stresses, strains and strain energy densities in a much smaller FE model, consisting of 1497 elements, representing a typical part of $20 \times 20 \times 20$ voxels taken from the trabecular structure. This model was analyzed using both the MARC FE code (MARC Analysis Corporation, Palo Alto, CA) and the methods described here. The results using the newly developed EBE strategy compared exactly to those found using the MARC FE code within the accuracy of the computer. The validity of the algorithms for larger problems was checked by simulating unconfined compression experiments on solid $\left(V_{v}=1\right)$ square cube models of up to $100 \times 100 \times 100$ elements. The results matched the analytical solution for this problem.

Boundary conditions of the FE model were varied to represent the situation in a compressive-test setup first, and in in situ conditions later. In the compressive-test model, a displacement $u_{\mathrm{p}}$ of $-0.0512 \mathrm{~mm}$ was prescribed to the top face (in the $z$-direction), equivalent to $1 \%$ strain for the cube as a whole. At the bottom face the displacements in the z-direction were constrained. All other faces of the cube were unconstrained (Fig. 3). This case simulates a compression test on the cube, with zero friction between the test platens and the bone. Hence, the cube is in a state of uniaxial stress at the apparent level. In the in situ model, the displacements of the other faces were suppressed as well, but only in the directions perpendicular to the cup faces (Fig. 3). Linde and Hvid (1989) demonstrated that the increase in stiffness due to these rigid side-contraints is in the same order of magnitudes as found in situ where bone is side-constrained by surrounding bone. The same displacement $u_{\mathrm{p}}$ as in the compressive-test model was applied to the top face. So, in effect this model simulates confined compression conditions and a state of uniaxial strain on the apparent level. The loading axis, in this case, coincides with the longitudinal axis of the tibia, and the uniaxial displacement represents the displacement under the tibial plateau, due to articular loading.

All elements in the model were given isotropic material properties with an arbitrarily chosen Young's modulus of $1000 \mathrm{MPa}$ and a Poisson's ratio of 0.3 . Since the analysis is linear elastic, the results can be

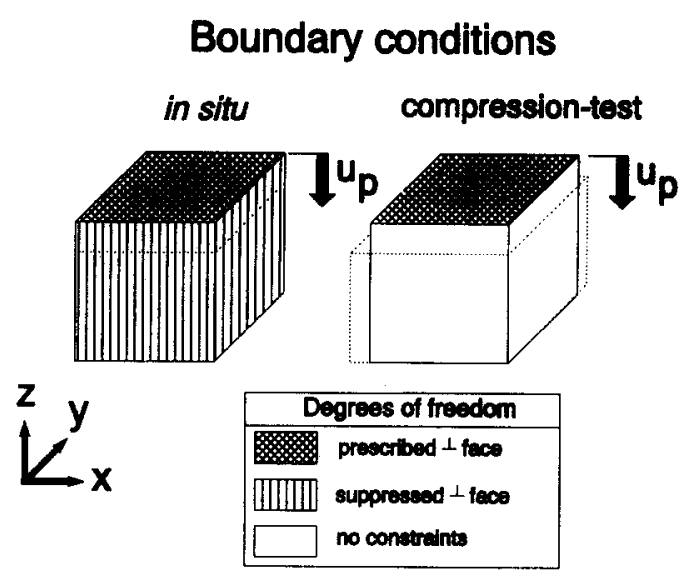

Fig. 3. The constraints applied to the in situ model (left) and the compression-test model (right). For both models a prescribed displacement $u_{\mathrm{p}}$ was applied at the top face while displacements were suppressed at the bottom face, but only in the direction perpendicular to this face.

scaled to any isotropic Young's modulus with the same Poisson's ratio.

Stresses, strains, Von Mises equivalent stresses and strain energy densities were calculated at tissue and at apparent levels. Tissue values will be presented in histograms as ratios between the tissue and the apparent value. To obtain more accurate results for the strain energy density, this value was calculated directly from the element nodal displacements and forces rather than from the element stresses and strains. The apparent strain energy density was calculated from the external nodal forces and the corresponding displacements.

The apparent Young's modulus in the z-direction for the specimen as a whole was calculated from the formula $E_{z}^{z}=\sigma_{z z}^{a} / \varepsilon_{z z}^{a}$, where $\sigma_{z z}^{a}$ is the apparent stress and $\varepsilon_{z z}^{2}$ the apparent strain in the $z$-direction. The apparent stress was calculated from $\sigma_{z z}^{\mathrm{z}}=F_{\mathrm{r}} /\left(h_{x} h_{y}\right)$, where $F_{\mathrm{r}}$ is the total reaction force at the top face, the apparent strain from $\varepsilon_{z z}^{z}=u_{\mathrm{p}} / h_{z}$, where $u_{\mathrm{p}}$ is the prescribed displacement; $h_{x}, h_{y}$ and $h_{z}$ represent the external dimensions of the cube in the $x-, y$ - and $z$ directions, respectively (Fig. 2). The apparent Young's modulus was calculated for the compressive-test model only; the set of boundary conditions for the in situ model will not allow for a proper apparent Young's modulus calculation.

The geometry of the FE model was such that the average individual trabecular cross-section as seen in the model was covered with at least four elements. A typical detail of the FE model (Fig. 4) shows the jagged boundaries that are present in the digitized mesh. To investigate possible inaccuracies in the calculations when using a digitized mesh, Hollister $e t$ al. (1992) used a two-dimensional model of a void structure that was analyzed using both a smooth and a digitized mesh. They found that the errors in the 
stiffness determination resulting from the digitization will be less than $10 \%$ for images with $50 \mu \mathrm{m}$ cubic voxels where the major structures are $100-200 \mu \mathrm{m}$ in thickness. To estimate the error due to digitization for realistic three-dimensional trabecular structures, a mesh convergence test was performed. For this test, another piece of trabecular bone of $2 \times 2 \times 2 \mathrm{~mm}$ was digitized using different resolutions. The voxel size was set to $20,40,60,80,100$ and $120 \mu \mathrm{m}$. The models thus obtained consisted of $321,341,38,658,11,551$, 4955, 2625 and 1271 elements, respectively. For each model the FE problem was solved and the apparent stiffness for the cube as a whole was calculated. The error in the tissue stress and strain distribution was assessed by comparing tissue stress and strain histograms for each model to those obtained for the model with the smallest voxel size.

\section{RESULTS}

The calculated apparent Young's modulus for the mesh convergence test models varied between 80 and $102 \mathrm{MPa}$ (Fig. 5). The maximum difference relative to the model with $20 \mu \mathrm{m}$ voxel size was $19 \%$ for the model with $100 \mu \mathrm{m}$ voxels, but no correlation was found between the apparent modulus and the voxel size for the range investigated. However, the variations in the modulus were correlated with differences in the volume fraction, which was not exactly the same in all models (varied between 0.309 and 0.328 ). It was concluded that for the apparent stiffness calculation the actual voxel size is not so important as long as the resulting FE model can describe the trabecular structure (in particular its volume fraction) reasonably well. The maximum differences between the tissue stress and strain histograms and the histograms for the $20 \mu \mathrm{m}$ model increased with increasing voxel size (Fig. 5). For the $40 \mu \mathrm{m}$ voxels used in the present study, the differences in the stress and strain histograms compared to a model with double that resolution will be less than 1.8 and $1.2 \%$, respectively. Accordingly, it was concluded that the $40 \mu \mathrm{m}$ voxel mesh was sufficiently converged.

The number of iterations required to solve the tibia sample FE model was dependent on the boundary conditions. For the in situ model, the conjugate gradient iterative solver used a total of 4119 iterations and $1.7 \mathrm{~h}$ CPU time on the CRAY-YMP computer, to obtain the solution within the accuracy interval. The compressive-test model required 10,082 iterations and $4.2 \mathrm{~h}$ CPU time. The ratio $\left\|f_{\text {res }}\right\| /\left\|f_{\text {reac }}\right\|$ was less than $3 E-6$ for both models.

At the apparent level, the total reaction forces were determined to be $-9.10 \mathrm{~N}$ for the in situ model and $-7.40 \mathrm{~N}$ in the compressive-test model (Table 1). The apparent stress calculated from these reaction forces is $-0.485 \mathrm{MPa}$ for the in situ model and $-0.394 \mathrm{MPa}$ for the compressive-test model. For the latter model an apparent Young's modulus of $39.4 \mathrm{MPa}$ was calculated. Assuming that the bone tissue is a homogeneous, isotropic and linear elastic material, its actual tissue Young's modulus can be estimated from the apparent Young's modulus, by scaling the calculated apparent modulus to an experimentally determined value. For a human proximal tibia, a range of realistic apparent Young's moduli as determined from experiments can be estimated from the apparent density using for instance the formulas given by Hodgkinson and Currey (1992): $\left(E^{a}\right)_{\text {low }}=10^{(-4.10+2.47 \log p)}$ for the lowest, $\left(E^{\mathrm{a}}\right)_{\text {mean }}=10^{(-2.43+1.96 \log \rho)}$ for the mean, and $\left(E^{\mathrm{a}}\right)_{\mathrm{high}}=10^{(-1.46+1.66 \log \rho)}$ for the highest experimentally established Young's modulus, where $\rho$ represents the apparent density. In the present example an approximated value for the apparent density is $280 \mathrm{~kg} \mathrm{~m}^{-3}$ (approximated from the volume fraction of 0.14 and a bone tissue density of $2.0 \mathrm{E} 3 \mathrm{~kg} \mathrm{~m}^{-3}$ ). Using the above formulas, a lowest apparent Young's modulus of $88.0 \mathrm{MPa}$ was calculated, a mean value of $233 \mathrm{MPa}$ and a highest apparent Young's modulus of $400 \mathrm{MPa}$. The apparent Young's modulus calculated for the compression-test model was $39.4 \mathrm{MPa}$, using a tissue modulus of $1000 \mathrm{MPa}$. Since the stresses and strains are linearly related to the tissue modulus in the model, it can be established that a tissue modulus of $2.23 \mathrm{GPa}$ would give exactly the lowest apparent modulus value, a tissue value of $5.91 \mathrm{MPa}$ would give the mean apparent modulus value, and a tissue modulus of $10.1 \mathrm{GPa}$ would give the highest apparent modulus.

At the microstructural level, stresses and strains in the trabeculae were calculated from the FE results for the in situ model. It was found that the strains in the trabeculae, shown in Fig. 6, are generally much less than the apparent value $\varepsilon_{z z}^{a}$; few elements have values that exceed the apparent value. Some ratios exceed 1.0, but these are not shown in the graphs of Fig. 6; the maximal ratio of tissue and apparent strain found was 50.8 for the $\varepsilon_{z z}^{t}$ component. The stress distribution, shown in Fig. 7, is wider than the tissue strain distribution because the tissue stresses are generally higher than the apparent stress. The maximal tissue-to-apparent stress ratio was 115.0 for the $\sigma_{z z}^{\mathrm{i}}$ component. However, few elements have values that exceed 20 times the apparent value. The mean tissue-to-apparent ratio $\bar{\sigma}_{z z}^{t} / \sigma_{z z}^{a}$ was 7.07 . For the Von Mises equivalent stress (Fig. 8) the mean ratio between the tissue and the apparent values was 10.7 , the maximal tissue-to-apparent ratio was 144.3. The strain energy density distribution at tissue level (Fig. 8) is the widest, with a maximal ratio of 1029 times the apparent strain energy density. The mean ratio of tissue and apparent strain energy density was $\bar{U}^{\prime} / U^{a}=7.07$. A contour plot of the tissue strain energy density distribution for a ten-element thick cross-section taken from the middle of the cube is shown in Fig. 9. The distribution shows relatively high loaded regions inside trabeculae oriented in the overall load direction. 
Apparent modulus

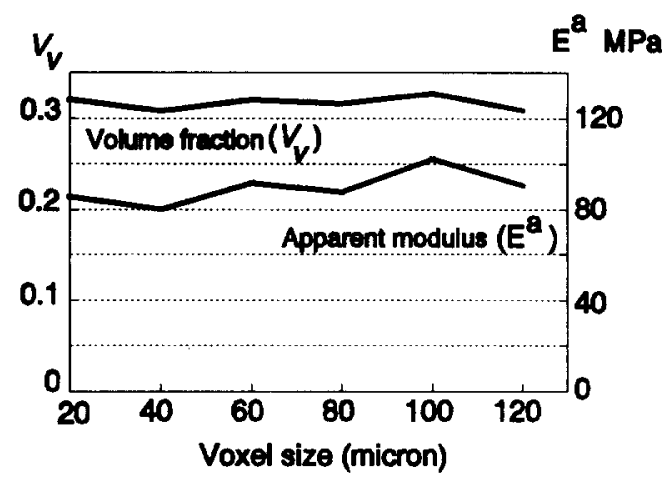

Tissue stress and strain histograms

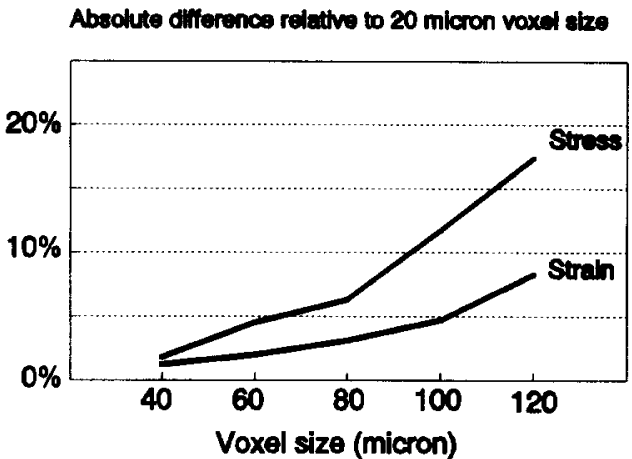

Fig. 5. Results of the mesh convergence test: on the left the calculated apparent modulus as a function of the voxel size; on the right the absolute differences (in percent) between the stress and strain histograms for the $20 \mu \mathrm{m}$ model when compared to the other models.

Table 1. The apparent values calculated for both models; the displacement at the top and thus the strain in the $z$-direction were prescribed

\begin{tabular}{lll}
\hline & In situ model & Compressive-test model \\
\hline Displacement top & $u_{\mathrm{p}}=-0.0512 \mathrm{~mm}$ & $u_{\mathrm{p}}=-0.0512 \mathrm{~mm}$ \\
Total force top & $F_{\mathrm{r}}=-9.10 \mathrm{~N}$ & $F_{\mathrm{r}}=-7.40 \mathrm{~N}$ \\
Strain $z$-direction & $\varepsilon_{z z}^{\mathrm{a}}=-0.01$ & $\varepsilon_{z z}^{\mathrm{a}}=-0.01$ \\
Stress $z$-direction & $\sigma_{z z}^{\mathrm{a}}=-0.485 \mathrm{MPa}$ & $\sigma_{z z}^{\mathrm{a}}=-0.394 \mathrm{MPa}$ \\
Strain energy density & $U^{\mathrm{a}}=0.242 \mathrm{E}-02 \mathrm{~N} \mathrm{~mm}^{-2}$ & $U^{\mathrm{a}}=0.197 \mathrm{E}-02 \mathrm{~N} \mathrm{~mm}^{-2}$ \\
\hline
\end{tabular}

\section{Tissue strain distributions in situ model}
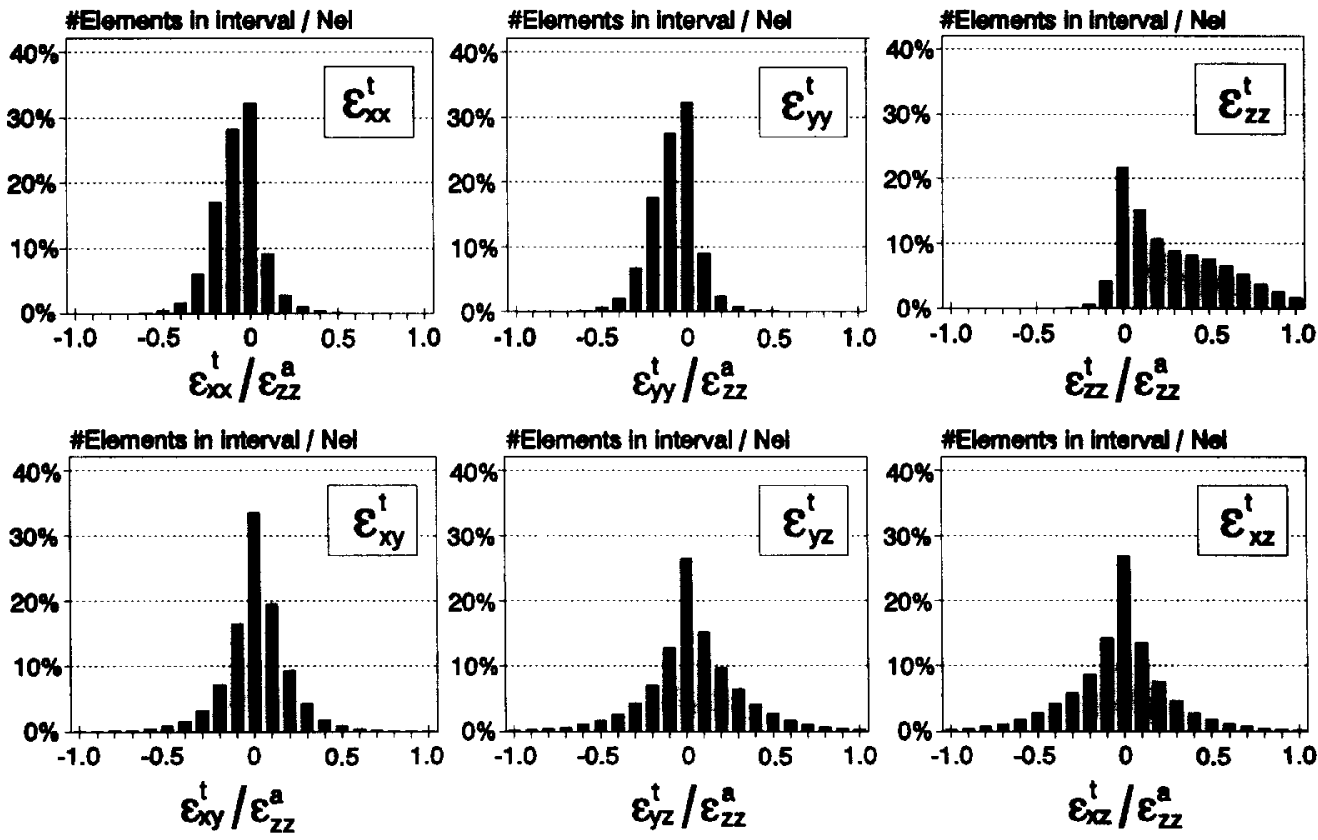

Fig. 6. Histograms showing the distribution of the tissue strain components in the in situ model. On the horizontal axis the ratio between the tissue strain component and the longitudinal apparent strain is indicated, on the vertical axis the number of elements in a bin as a percentage of the total number of elements Nel. The bin width is set to $0.1 \varepsilon_{z z}^{z}$. Note that the apparent strain is the same in all histograms. 


\section{Tissue stress distributions}

\section{in situ model}
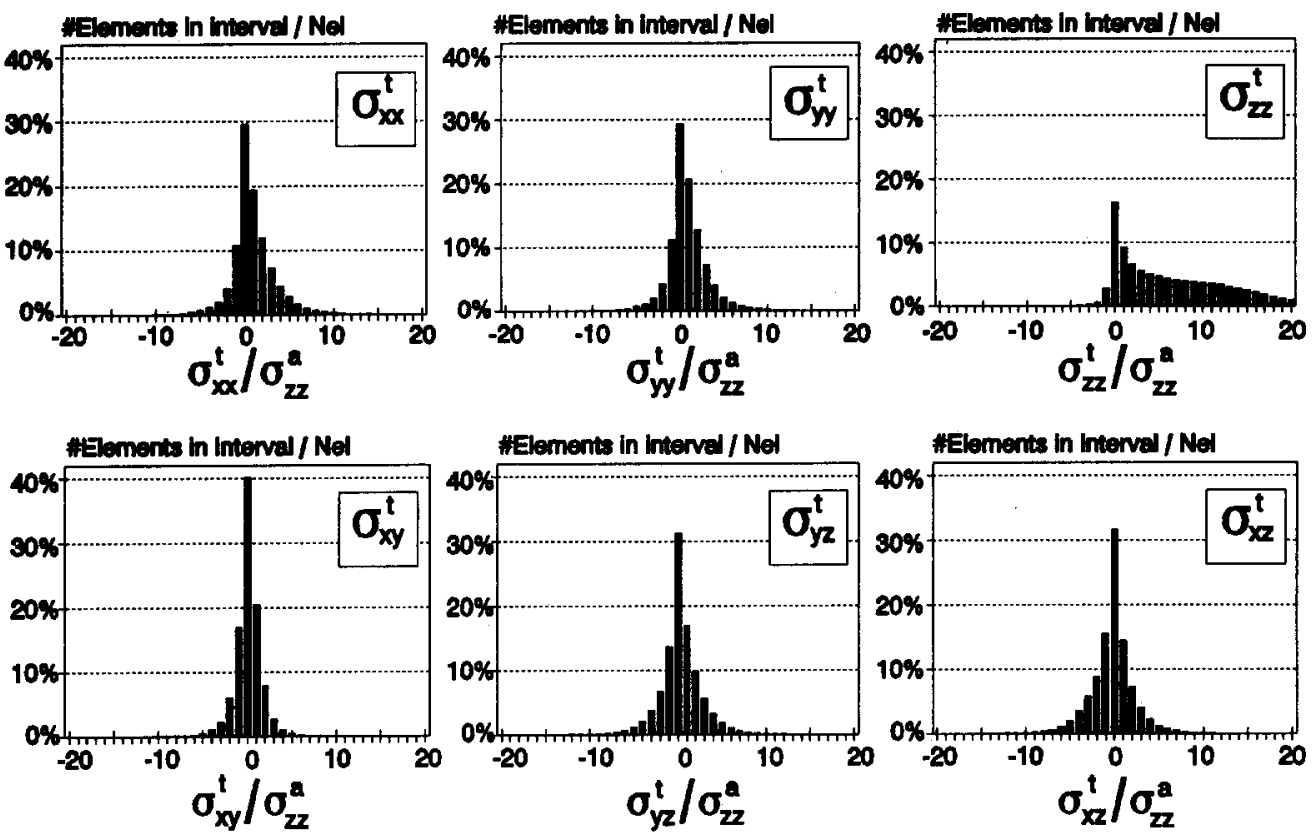

Fig. 7. Histograms showing the distribution of the tissue stress components in the in situ model. On the horizontal axis the ratio between the tissue stress component and the longitudinal apparent stress is indicated, on the vertical axis the number of elements in a bin as a percentage of the total number of elements Nel. The bin width is set to $1.0 \sigma_{2 z}^{\mathrm{a}}$. Note that the apparent stress is the same in all histograms.

\section{Strain energy density}

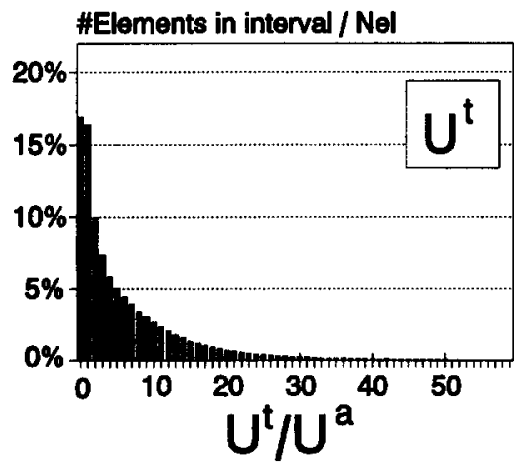

Von Mises

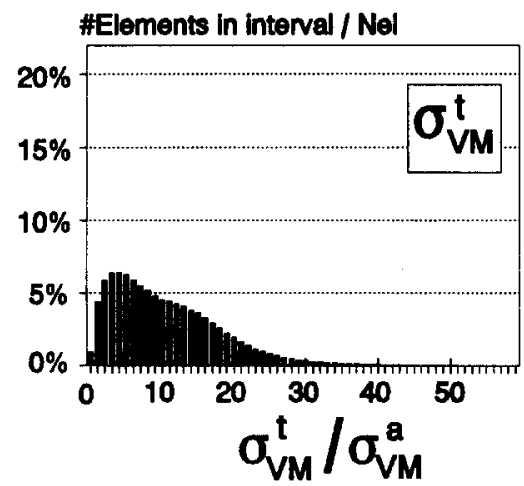

Fig. 8. Histograms showing the distribution of the tissue strain energy density and the tissue Von Mises equivalent stress in the in situ model. On the horizontal axis the ratio between the tissue component and the corresponding apparent value is indicated, on the vertical axis the number of elements in a bin as a percentage of the total number of elements Nel. The bin width is set to $1.0 U^{\mathrm{a}}$ for the strain energy density distribution and to $1.0 \sigma_{\mathrm{V}}^{\mathrm{N}}$ for the Von Mises distribution.

\section{DISCUSSION}

In the present paper we have introduced a new approach to analyze trabecular bone structures using large-scale FE models, constructed from voxel data. By using the fact that all elements in the resulting FE model are the same, it became possible to analyze a piece of trabecular bone that is large enough (of the size of a test sample) to obtain relevant apparent results (Harrigan et al., 1988). Using this model, it is possible to estimate the tissue stress and strain distribution at any location in the bone. In addition, it is 
possible to estimate the tissue material properties as functions of the apparent material properties.

Some limitations of the present model have to be discussed. First, the model has a smallest dimension of $3.64 \mathrm{~mm}$. It can be questioned if this is large enough for a continuum assumption. Harrigan et al. (1988) have determined that averaging over five trabecular lengths should provide sufficiently average continuum quantities. From the cross-section shown in Fig. 9 it can be estimated that this condition is met for the present sample size. Second, the accuracy of the tissue stress and strain calculation for the $40 \mu \mathrm{m}$ voxel size used in the present model was investigated by comparing the calculated histograms for digitized models with different resolution. It should be noted that this mesh convergence test cannot be used to investigate the error in the tissue stress and strain values at a specific boundary location. Jacobs et al. (1993) have shown that tissue strains at the boundaries of a digitized mesh can differ substantially from those found for a smooth mesh, and that this error is not necessarily reduced when the element size is reduced. However, the error in the histograms will be reduced with smaller elements, because the fraction of elements in the jagged area is reduced, and thus the accuracy of the histograms is verified. Third, the exact in situ boundary conditions for the present bone sample are unknown. It is possible that realistic in situ conditions would allow for some more deformation at the sides of the specimen than the confined compression condition imposed at the in situ model used in the present study. To check for this, tissue strain histograms were also determined for the compressive-test model, which allows for more deformation than the in situ situation. It was found that the histograms for the compressive-test model are very similar to those shown in Fig. 6, and thus that the actual conditions at the side of the cube hardly affect the conclusions.

In the present study we used average experimental values to calculate a realistic range for the tissue Young's modulus: $2.23-10.1 \mathrm{GPa}$. A mean value of $5.91 \mathrm{MPa}$ was calculated. This value is in good agreement with the experimentally established trabecular tissue modulus in the proximal tibia of $4.59 \mathrm{GPa}$ found by Choi et al. (1990) and of $5.72 \mathrm{GPa}$ found by Choi and Goldstein (1992). However, the comparison between direct measurements on small specimens and the values as determined in the present study can be misleading. The small size of single trabeculae specimens and damage effects due to machine cutting of small specimens will likely lead to an underestimation of the bulk tissue modulus. The indirect measurement method presented here does not suffer from these effects, and thus a significant higher tissue modulus would be expected. The fact that a higher value is not found in the present study can possibly be explained by systematic errors in the determination of the apparent modulus in compression experiments. Odgaard and Linde (1991) and Keaveny et al. (1993) have demonstrated that artifacts at the bone-platen interface may result in a substantial underestimation of the experimentally established apparent moduli and thus of the tissue modulus as determined in the present study. Therefore it is expected that a realistic tissue modulus is closer to the upper boundary of the range determined.

Although not the aim of the present study, the methods presented here can be used to exclude the damage artifact error. This can be done by creating a full size model of a specimen that was experimentally tested, thereby also modeling the damaged zone with platen-specimen boundary conditions chosen exactly as in the experiment. Such full size models can also be used to determine material properties that are nonlinear functions of the deformations, such as the Poisson's ratios.

At the tissue level, the ratios between the mean tissue stress and the apparent stress in the load direction and between the mean tissue and the apparent strain energy density must equal $1 / V_{v}$, where $V_{v}$ is the volume fraction of the cube (Appendix A). Indeed it was found in the present analysis that $\bar{\sigma}_{z z}^{\mathrm{t}} / \sigma_{z z}^{\mathrm{a}}=7.07$ and $\bar{U}^{t} / U^{\mathrm{a}}=7.07$, which is precisely equal to $1 / V_{v}$ for $V_{v}=0.14$ in the present model. In fact, this result confirms the validity of the stress and strain energy density calculation for the present FE model. Carter et al. (1987) have introduced the mean tissue strain energy density in the bone tissue $U^{\mathrm{a}} / V_{v}$ as the objective function for bone remodeling. The present analysis shows that the actual strain energy density in the trabeculae can be much higher (up to $1029 V_{v}=144$ times higher) than the mean tissue strain energy density. Since the effect of mechanically induced bone remodeling is considered at the apparent level (by the change in apparent density) the actual distribution of the objective function may be of lesser importance. However, for processes that are initiated by the maximum tissue strain energy density or maximum tissue effective stress, for example microdamage processes, the mean tissue value is not a good measure. The ratio between the mean tissue Von Mises equivalent stress and the apparent Von Mises stress, $\bar{\sigma}_{\mathrm{VM}}^{\mathrm{A}} / \sigma_{\mathrm{VM}}^{\mathrm{a}}$, does not equal $1 / V_{v}$, indicating that the mean tissue Von Mises stress cannot be estimated from the apparent Von Mises stress divided by the volume fraction (Appendix A).

From the tissue stress and strain distributions as shown in Figs 6 and 7, a number of conclusions can be derived. First, it can be seen that the axial deformation in the tissue material $\left(\varepsilon_{z z}^{t}\right)$ is smaller than the apparent deformation $\left(\varepsilon_{z z}^{a}\right)$; very few elements have a tissue-to-apparent ratio greater than 1.0 (Fig. 6). Gibson (1985) has shown that this result is typical for asymmetric structures, in which the deformation mode is in bending rather than in axial deformation. From this, it can be concluded that the trabecular structure investigated in the present study can be characterized as an asymmetric structure rather than a columnar one. Due to bending effects, trabeculae can be tensile strained under compressive apparent loading (Fig. 10), resulting in tissue-to-apparent stress 


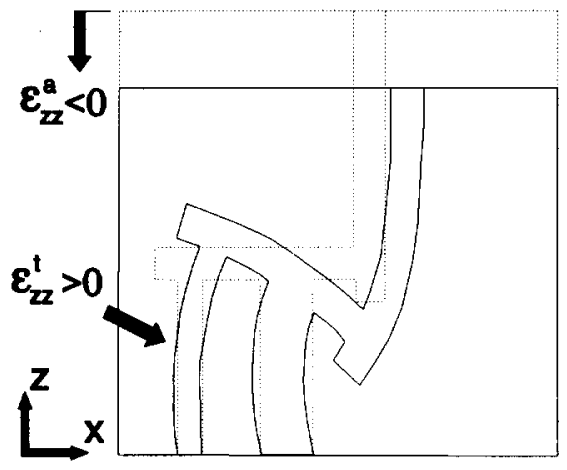

Fig. 10. Tissue tensile strains $\left(\varepsilon_{z z}^{t}>0\right)$ can occur under compressive apparent loading $\left(\varepsilon_{z z}^{a}<0\right)$ due to bending effects.

and strain ratios in the load direction which are less than zero. From Figs 6 and 7 it can be seen that such deformation modes are indeed present in the structure. This paradoxical effect of trabeculae being tensile strained under compressive apparent loading was found earlier by Odgaard and Linde (1991), when using optical strain measurement on the individual trabeculae. Second, for the bin width chosen, the stress distributions show a peak at zero for all components, i.e. at many locations in the tissue the individual stress components are very small. The Von Mises stress distribution, however, shows no high peak at zero. This indicates that at most locations the elements are distorted, thus implying that most trabeculae are load carrying. High tissue-to-apparent ratios are found for the Von Mises stress and for the strain energy density, although the number of elements with excessive values is relatively small. It is possible that a more physiological loading condition (for instance obtained from multiaxial load cases) will reduce the maximal Von Mises and strain energy density values.

The applications of the new method discussed here are, of course, only examples of its applicability. Other examples are the investigation of various bench tests for trabecular bone by simulating the test conditions. For example, the boundary effects in compressive tests can be evaluated. Secondly, the method can be useful to estimate tissue stresses in vivo, in various circumstances and locations. It can be used to study damage-accumulation processes by successively eliminating elements that are overloaded from the model, and bone remodeling processes at the tissue level. Finally, it can be applied as a link between mechanics at the whole-bone level and the microscopic level.

Acknowledgements-This project was sponsored by The Netherlands Foundation for Research (NWO/Medical Sciences), by the National Computing Facilities Foundation (NCF), and by the Danish Rheumatism Association (233- 588). The authors gratefully acknowledge the selection of this paper for the Selvik Award by the European Orthopaedic Research Society (EORS), London, 11 April, 1994.

\section{REFERENCES}

Beaupré, G. S. and Hayes, W. C. (1985) Finite element analysis of a three-dimensional open-celled model for trabecular bone. J. biomech. Engng 107, 249-256.

Carter, D. R., Fyhrie, D. P. and Whalen, R. T. (1987) Trabecular bone density and loading history: regulation of connective tissue biology by mechanical energy. J. Biomechanics 20, 785-794.

Choi, K. and Goldstein, S. A. (1992) A comparison of the fatigue behavior of human trabecular and cortical bone tissue. J. Biomechanics 25, 1371-1381.

Choi, K., Kuhn, J. L., Ciarelli, M. J. and Goldstein, S. A. (1990) The elastic moduli of human subchondral, trabecular, and cortical bone tissue and the size dependency of cortical bone modulus. J. Biomechanics 23, 1103-1113.

Edidin, A. A., Dawson, J. M., Zhu, M. and Chinchalkar, S. (1993) Direct estimation of the modulus of cancellous bone using a variable-stiffness FE model. Trans. 39th A. Meeting Orthop. Res. Soc., p. 589.

Feldkamp, L. A., Goldstein, S. A., Parfitt, A. M., Jesion, G. and Kleerekoper, M. (1989) The direct examination of three dimensional bone architecture in vitro by computed tomography. J. Bone Min. Res. 4, 3-11.

Frost, H. M. (1987) Vital biomechanics. Proposed general concepts for skeletal adaptations to mechanical usage. Calcif. Tissue Int. 42, 145-156.

Fyhrie, D. P. and Hamid, M. S. (1993) The probability distribution of trabecular level strains for vertebral cancellous bone. Trans. 39th A. Meeting Orthop. Res. Soc., p. 175.

Fyhrie, D. P., Hamid, M. S., Kuo, R. F. and Lang, S. M. (1992) Direct three-dimensional finite element analysis of human vertebral cancellous bone. Trans. 38th A. Meeting Orthop. Res. Soc., p. 551.

Gibson, L. J. (1985) The mechanical behavior of cancellous bone. J. Biomechanics 18, 317-328.

Harrigan, T. P., Jasty, M., Mann, R. W. and Harris, W. H. (1988) Limitations of the continuum assumption in cancellous bone. J. Biomechanics 21, 269-275.

Hayes, L. J. and Devloo, P. (1986) A vectorized version of a sparse matrix-vector multiply. Int. J. Numer. Methods Engng 23, 1043-1056.

Hodgkinson, R. and Currey, J. D. (1992) Young's modulus, density and material properties in cancellous bone over a large density range. J. Mater. Sci. Mater. Med. 3, 377-381.

Hollister, S. J., Brennan, J. M. and Kikuchi, N. (1992) Recent Advantages in Computer Methods in Biomechanics and Biomedical Engineering, pp. 308-317. Books and Journals Int. Ltd. Swansea, U.K.

Hollister, S. J., Fyhrie, D. P., Jepsen, K. J. and Goldstein, S. A. (1991) Application of homogenization theory to the study of trabecular bone mechanics. J. Biomechanics 24, 825-839.

Hollister, S. J. and Kikuchi, N. (1992) Direct analysis of trabecular bone stiffness and tissue level mechanics using an element-by-element homogenization method. Trans. 38th A. Meeting Orthop. Res. Soc., p. 559.

Hughes, J. R., Ferencz, R. M. and Hallquist, J. O. (1987) Large-scale vectorized implicit calculations in solid mechanics on a cray X-MP/48 utilizing EBE preconditioned conjugate gradients. Comput. Methods appl. Mech. Engng 61, 215-248.

Jacobs, C. R., Mandell, J. A. and Beauprè, G. S. (1993) A comparative study of automatic finite element mesh generation techniques in orthopaedic biomechanics. Bioengng Conf. ASME, BED-24, 512-514.

Keaveny, T. M., Borchers, R. E., Gibson, L. J. and Hayes, W. C. (1993) Theoretical analysis of the experimental artifact in trabecular bone compressive modulus. J. Biomechanics 26, 599-607.

Kuhn, J. L., Goldstein, S. A., Choi, K. W., London, M., Feldkamp, L. A. and Matthews, L. S. (1989) Comparison 
of the trabecular and cortical tissue moduli from the human iliac crests. J. orthop. Res. 7, 876-884.

Linde, F. and Hvid, I. (1989) The effect of constraint on the mechanical behaviour of trabecular bone specimens. J. Biomechanics 22, 485-490.

Martin, R. B. and Burr, D. B. (1989) Structure, Function and Adaptation of Compact Bone. Raven Press, New York.

Odgaard, A., Andersen, K., Melsen, F. and Gundersen, H. J. G. (1990) A direct method for fast three-dimensional serial reconstruction. J. Microscopy 159, 335-342.

Odgaard, A. and Linde, F. (1991) The underestimation of Young's modulus in compressive testing of cancellous bone specimens. J. Biomechanics 24, 691-698.

Rho, J. Y., Ashman, R. B. and Turner, C. H. (1993) Young's modulus of trabecular and cortical bone material: ultrasonic and microtensile measurements. J. Biomechanics $\mathbf{2 6}$, 111-119.

Ryan, J. C. and Williams, J. L. (1989) Tensile testing of rodlike trabeculae excised from bovine femoral bone. J. Biomechanics 22, 351-355.

Strang, G. (1986) Introduction to Applied Mathematics. Wellesley-Cambridge Press, Wellesley, Massachusetts.

Williams, J. L. and Lewis, J. L. (1982) Properties and an anisotropic model of cancellous bone from the proximal tibia epiphysis. J. biomech. Engng 104, 50-56.

\section{APPENDIX A}

\section{RATIO BETWEEN MEAN TISSUE AND APPARENT VALUE FOR STRESSES AND STRAIN ENERGY DENSITY}

The ratio for the mean tissue to apparent stress in the load direction can be determined for a cube with no constraints in the load direction at the sides of the cube. The average tissue stress in the $z$-direction equals

$$
\overline{\sigma_{z z}^{\mathrm{t}}}=\frac{1}{V^{\mathrm{t}}} \int \sigma_{z z}^{\mathrm{t}} \mathrm{d} V^{\mathrm{t}}=\frac{1}{V^{\mathrm{t}}} \iint \sigma_{z z}^{\mathrm{t}} \mathrm{d} A^{\mathrm{t}} \mathrm{d} z,
$$

where $V^{t}=V_{v} V^{a}$ is the tissue volume as a function of the volume fraction $V_{v}$ and the apparent volume $V^{2}$, and $A^{1}=V_{\mathrm{S}} A$ is the tissue area at a cross-section perpendicular to the load direction, with a surface fraction $V_{\mathrm{s}}$. The total apparent force $F_{z}$ in the $z$-direction must balance the total tissue force in that direction in each perpendicular crosssection:

$$
F_{z}=\int \sigma_{z z}^{\mathrm{t}} \mathrm{d} A^{\mathrm{t}}
$$

From equations (1) and (2) it follows that

$$
\overline{\sigma_{z z}^{\mathrm{t}}}=\frac{1}{V^{\mathrm{t}}} \int F_{z} \mathrm{~d} z=\frac{h_{z} F_{z}}{V^{\mathrm{t}}}=\frac{V^{\mathrm{a}} F_{z}}{V^{\mathrm{t}} A}=\frac{1}{V_{v}} \sigma_{z z}^{\mathrm{a}},
$$

where $h_{z}$ is the size of the cube in the $z$-direction. The same ratio holds for the stress component in the $x$ - and $y$-directions as long as the equilibrium equation (2) holds in each cross-section perpendicular to the corresponding direction. For the Von Mises equivalent stress, however, such an equilibrium equation, in general, cannot be found, and therefore the average tissue Von Mises equivalent stress cannot be determined from the apparent value and the volume fraction.

For the strain energy density another equilibrium equation can be used based on the total apparent energy $V^{a} U^{a}$ that must equal the total internal energy in the tissue material $V^{t} \bar{U}^{t}$, where $\bar{U}^{\mathrm{t}}$ is the average tissue strain energy density; thus

$$
\bar{U}^{\prime}=\frac{V^{\mathrm{a}}}{V^{\mathrm{t}}} U^{\mathrm{a}}=\frac{1}{V_{v}} U^{\mathrm{a}}
$$

